Маркеры острого

воспаления у пашиентов

c COVID-19 в сочетании

с гипертонической болезнью

\author{
Киселева В.В., \\ Ячменев К.С., \\ Зайцева А.Ю.
}

\begin{abstract}
Федеральное государственное бюджетное образовательное учреждение высшего образования "Курский государственный медицинский университет" Министерства зАравоохранения Российской Федерации, 305041, г. Курск, Российская Федерация
\end{abstract}

Гипертоническая болезнь - наиболее распространенное сопутствующее заболевание при COVID-19. Хотя повышенная смертность от новой коронавирусной инфекции, протекающей на фоне сопутствующей гипертонической болезни, доказана многократно, большей проблемой является поиск прогностических факторов, которые бы позволили на ранних этапах прогнозировать риск развития тяжелого течения инфекции, вызванной SARS-CoV-2, и летального исхода.

Цель исследования - определение уровней некоторых маркеров острого воспаления [ферритин, С-реактивный белок (СРБ), интерлейкин-6 (ИЛ-6)] у пациентов с новой коронавирусной инфекцией, сочетающейся с гипертонической болезнью.

Материал и методы. В исследование включены 130 пациентов 2 групп: основная группа - пациенты с COVID-19, протекавшей на фоне гипертонической болезни ( $n=70)$, контрольная группа - пациенты с COVID-19 средней степени тяжести течения без сопутствующих заболеваний $(n=60)$. Критерии включения в основную группу: наличие гипертонической болезни II стадии (медикаментозно контролируемой) и отсутствие дополнительных сопутствующих заболеваний, включая выраженное ожирение $\geq$ II степени (индекс массы тела $\geq 35$ кг $/ \mathrm{m}^{2}$ ). Пациенты основной группы по возрасту (от 38 до 65 лет, средний возраст 57,0 6,9 года) не отличались от пациентов контрольной группы (от 42 до 65 лет, средний возраст 53,6 6,6 года). Женщины преобладали в обеих группах: $61,4 \%$ в основной группе и 70\% - в контрольной. Критериями исключения пациентов были частота дыхательных движений $\leq 30$ в минуту, насыщение крови кислородом $\left(\mathrm{SpO}_{2}\right)$ не ниже 93\%, отсутствие положительного результата исследования на PHK SARS-CoV-2 методом полимеразной цепной реакции из носо- и ротоглотки, изменения на компьютерной томограмме (КТ), характерные для вирусного поражения, с вовлечением более 50\% паренхимы легкого (КТ 3-4), показатели уровня ИЛ-6 $>40$ пг/мл, возраст старше 65 лет.

Результаты. Показатель ферритина >500 нг/мл был у 30\% больных основной и 23,3\% контрольной группы. Сравнение уровня ферритина в 2 группах показало, что его показатели у пациентов основной группы были достоверно выше аналогичных показателей контрольной группы $(p=0,033 ; p<0,05)$. СРБ также чаще был повышен у пациентов основной группы с артериальной гипертензией, чем у больных без гипер-

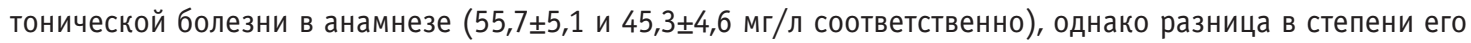
повышения недостоверна $(p=0,132 ; p>0,05)$. Среднее значение ИЛ-6 в обеих группах находилось в пре-

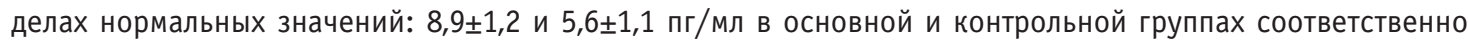
$(p=0,045 ; p<0,05)$.

Заключение. У пациентов с COVID-19, протекавшим на фоне гипертонической болезни, происходило достоверное возрастание уровня ряда маркеров острого воспаления (ферритин, ИЛ-6) по сравнению с пациентами без сопутствующей артериальной гипертензии. Данные изменения могут быть связаны с влиянием повышенного артериального давления на сосудистую стенку, а также с тропностью вируса к эндотелиальным клеткам сосудов.
Ключевые слова: COVID-19, гипертоническая болезнь, маркеры воспаления, ферритин

Финансирование. Исследование не имело спонсорской поддержки.

Конфликт интересов. Авторы заявляют об отсутствии конфликта интересов.

Вклад авторов. Концепция и дизайн исследования - Киселева В.В.; сбор и обработка материала - Киселева В.В., Зайцева Л.Ю., Ячменев К.С.; статистическая обработка, написание текста - Ячменев К.С.; редактирование, утверждение окончательного варианта статьи - Киселева В.В. 
Для цитирования: Киселева В.В., Ячменев К.С., Зайцева Л.Ю. Маркеры острого воспаления у пациентов с COVID-19 в сочетании с гипертонической болезнью // Инфекционные болезни: новости, мнения, обучение. 2021. T. 10, № 4. C. 22-28. D0I: https:// doi.org/10.33029/2305-3496-2021-10-4-22-28

Статья поступила в редакцию 17.04.2021. Принята в печать 01.10.2021.

\section{Markers of acute inflammation in patients with COVID-19 in combination with hypertension}

Kiseleva V.V., Yachmenev K.S.

Zaitseva L.Yu.
Kursk State Medical University of the Ministry of Healthcare of the Russian Federation, 305041, Kursk, Russian Federation

Hypertension is the most common concomitant disease in COVID-19. Although the increased mortality from a new coronavirus infection occurring against the background of concomitant hypertension has been proven many times, the greater problem is the search for prognostic factors that would allow predicting the risk of severe infection caused by SARS-CoV-2 and death at an early stage.

The aim of the study was to determine the level of some markers of acute inflammation (ferritin, C-reactive protein, IL-6) in patients with a new coronavirus infection combined with hypertension.

Material and methods. The study included 130 patients of two groups: the main group - patients with COVID-19, occurring against the background of hypertension $(n=70)$, the control group - patients with COVID-19 of moderate severity without concomitant diseases $(n=60)$. Criteria for inclusion in the main group: the presence of stage II hypertension (drug-controlled) and the absence of additional concomitant diseases, including severe obesity $\geq$ II degrees (body mass index $\geq 35 \mathrm{~kg} / \mathrm{m}^{2}$ ). Patients of the main group by age (from 38 to 65 years, average age $57.0 \pm 6.9$ years) did not differ from patients of the control group (from 42 to 65 years, average age $53.6 \pm 6.6$ years). Women prevailed in both groups $-61.4 \%$ in the main group and $70 \%$ in the control group, respectively. The criteria for excluding patients were: $\mathrm{BDD}$ no more than $30 / \mathrm{min}, \mathrm{SpO}_{2}$ no lower than $93 \%$, absence of a positive result of a study on SARS-CoV-2 RNA by PCR from the nasopharynx and oropharynx, CT changes characteristic of a viral lesion involving more than $50 \%$ of lung parenchyma (CT 3-4), IL-6 levels over $40 \mathrm{pg} / \mathrm{ml}$, age over 65 years.

Results. The ferritin index above $500 \mathrm{ng} / \mathrm{ml}$ was in $30 \%$ of patients of the main and $23.3 \%$ of the control group. Comparison of ferritin levels in the two groups showed that its indicators in patients of the main group were significantly higher than those of the control group $(p=0.033 ; p<0.05)$. C-reactive protein (CRP) was also more often elevated in patients of the main group with arterial hypertension than in patients without a history of hypertension $(55.7 \pm 5.1$ and $45.3 \pm 4.6 \mathrm{mg} / \mathrm{l}$, respectively), but the difference in the degree of its increase was not significant $(p=0.132 ; p>0.05)$. The average value of IL-6 in both groups was within normal values: $8.9 \pm 1.2 \mathrm{pg} / \mathrm{ml}$ in the main group and $5.6 \pm 1.1 \mathrm{pg} / \mathrm{ml}$ in the control group $(p=0.045 ; p<0.05)$.

Conclusion. In patients with COVID-19, which occurred against the background of hypertension, there was a significant increase in the level of a number of markers of acute inflammation (ferritin, IL-6) compared with patients without concomitant hypertension. These changes may be associated with the effect of high blood pressure on the vascular wall, as well as with the tropicity of the virus to vascular endothelial cells.

Funding. The study had no sponsor support.

Conflict of interest. The authors declare no conflict of interest.

Contribution. Concept and design of the study - Kiseleva V.V.; collection and processing of the material - Kiseleva V.V., Zaitseva L.Yu., Yachmenev K.S.; statistical processing, text writing - Yachmenev K.S.; editing, approval of the final version of the article - Kiseleva V.V.

For citation: Kiseleva V.V., Yachmenev K.S., Zaitseva L.Yu. Markers of acute inflammation in patients with COVID-19 in combination with hypertension. Infektsionnye bolezni: novosti, mneniya, obuchenie [Infectious Diseases: News, 0pinions, Training]. 2021; 10 (4): 22-8. D0I: https://doi.org/10.33029/2305-3496-2021-10-4-22-28 (in Russian)

Received 17.04.2021. Accepted 01.10.2021.

$\mathrm{H}$ овая коронавирусная инфекция, вызванная вирусом SARS-CoV-2 (COVID-19), стала настоящим испытанием для человечества. За год пандемии изучены многие аспекты патогенеза, клинических проявлений, диагностики и лечения COVID-19, что позволило спасти множество жизней, однако некоторые аспекты до сих пор недостаточно известны. Одним из основных и значимых направлений изучения COVID-19 является прогнозирование течения заболевания у конкретного больного на основании определения
Keywords: COVID-19, hypertension markers of inflammation, ferritin 
Именно поэтому за ними необходим тщательный контроль в процессе лечения пациента [3].

Ферритин - неспецифичный маркер острого воспаления, обладающий антирадикальной функцией. Возрастание ферритина является компенсаторной реакцией организма, подавляющей повреждение клеточных элементов путем инактивации высокоактивного $\mathrm{Fe}^{2+}$, использующегося в клетках для окислительно-восстановительной реакции Фентона с образованием свободных радикалов и последующим повреждением белков, липидов и нуклеиновых кислот клетки, до интактного $\mathrm{Fe}^{3+}$. Ферритин изолирует свободное железо, предотвращая его участие в окислительно-восстановительных реакциях, в результате которых образуются свободные радикалы (преимущественно гидроксилы $\mathrm{OH}^{\circ}$ ) источники клеточного окислительного стресса; так, 1 молекула ферритина может депонировать около 4500 молекул железа [4].

Система синтеза ферритина напрямую связана с уровнем железа в клетке и регулируется железосвязывающим элементом (IRE) на 5'-конце мРНК и железорегулирующим белком (IRP). При низкой концентрации железа в клетке увеличивается сродство IRP к IRE, в результате трансляция белка на мРНК ингибируется и синтез ферритина замедляется. При возрастании уровня железа в клетке сродство IRP к IRE снижается и синтез ферритина увеличивается [4]. Помимо трансляционного уровня, синтез ферритина регулируется на уровне транскрипции: ряд провоспалительных цитокинов, например фактор некроза опухоли $\alpha$ и интерлейкин-1 $\beta$ (ИЛ-1 $\beta)$, способны индуцировать экспрессию Н-субъединиц ферритина. Однако имеются данные о том, что ферритин может индуцировать образование провоспалительных цитокинов [5].

Связь более тяжелого течения COVID-19 с наличием сопутствующих заболеваний обсуждается с самого возникновения пандемии. В силу широкой распространенности АГ и СД изучение их комбинации с инфекцией, вызванной SARSCoV-2, наиболее актуальна. Хотя повышенная смертность от новой коронавирусной инфекции, протекающей на фоне сопутствующей гипертонической болезни (ГБ), многократно доказана [6, 7], большую проблему представляет поиск прогностических факторов, которые позволили бы на ранних этапах прогнозировать риск тяжелого течения инфекции, вызванной SARS-CoV-2, и летального исхода. Особенно это актуально при наличии противоречивых данных, касающихся уровня повышения маркеров острой фазы (в частности ферритина) при сочетании COVID-19 и ГБ. S. Huang и соавт. утверждают, что при указанном сочетании заболеваний уровень ферритина ниже, чем при новой коронавирусной инфекции без сопутствующих заболеваний в анамнезе [8]. Однако в исследование были включены пациенты с различной степенью тяжести COVID-19, средний возраст пациентов в группе с сочетанием заболеваний был значительно выше, а различия уровня ферритина в крови статистически недостоверны $(p=0,288)$. В ряде исследований среди многих факторов, сопряженных с высокой летальностью пациентов с новой коронавирусной инфекцией, обособленно выделяют и повышенный уровень ферритина, и АГ без определения взаимосвязи двух этих факторов $[7,9]$. Более того, повы- шение уровня воспалительных белков в большинстве случаев непосредственно связано с инфекционным процессом и является неотъемлемой частью тяжелого течения COVID-19, отражая системный характер воспалительной реакции [10]. Напротив, J. Feld и соавт. считают, что ферритин не является надежным предиктором высокой летальности, однако главным критерием отбора пациентов в их исследование было повышение уровня ферритина, но связь уровня ферритина с сопутствующими заболеваниями не оценивали [11].

Цель исследования - определение уровня некоторых маркеров острого воспаления (ферритин, СРБ, ИЛ-6) у пациентов с новой коронавирусной инфекцией, сочетающейся с ГБ.

\section{Материал и методы}

Проведен ретроспективный анализ историй болезни пациентов, госпитализированных в ОБУЗ «ОКИБ» (Курск) с основным диагнозом «коронавирусная инфекция COVID-19 (подтвержденная), среднетяжелая форма. Осложнения: внебольничная двусторонняя пневмония, среднетяжелое течение» с августа по декабрь 2020 г. Диагноз подтвержден выявлением PHK SARS-CoV-2 методом полимеразной цепной реакции (тест-система «Ампли Тест SARS-CoV-2» ФГБУ «ЦСП» ФМБА России). Тяжесть течения COVID-19 определяли согласно Временным методическим рекомендациям по профилактике, диагностике и лечению новой коронавирусной инфекции (COVID-19) (далее - Рекомендации), существовавшим на момент госпитализации пациента (версии 7-9) [10].

Критерии исключения пациентов: частота дыхательных движений $\geq 30 /$ мин, насыщение крови кислородом $\left(\mathrm{SpO}_{2}\right)$ $\leq 93 \%$, отсутствие положительного результата исследования на PHK SARS-CoV-2 методом полимеразной цепной реакции из носо- и ротоглотки, изменения на компьютерной томограмме (КТ), характерные для вирусного поражения, с вовлечением более 50\% паренхимы легкого (КТ 3-4), показатели уровня ИЛ-6 $\geq 40$ пг/мл. Также в исследование не вошли пациенты старше 65 лет и с поражением паренхимы легких менее $10 \%$ по данным КТ.

В исследование включены 130 пациентов, которые были разделены на 2 группы. В основную группу вошли 70 пациентов с COVID-19 на фоне ГБ, в контрольную группу 60 пациентов с подтвержденным COVID-19 средней степени тяжести без сопутствующих заболеваний. Пациенты основной группы по возрасту (от 38 до 65 лет, средний возраст $57,0 \pm 6,9$ года) не отличались от пациентов контрольной

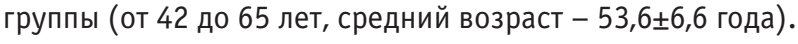

Критерии включения в основную группу: ГБ II стадии (медикаментозно контролируемая) и отсутствие дополнительных сопутствующих заболеваний, включая выраженное ожирение $\geq$ II степени [индекс массы тела (ИМТ) $\geq 35$ кг $/ \mathrm{m}^{2}$ ]. Выбор II стадии ГБ обусловлен поражением органов-мишеней в отсутствие ассоциированных с гипертензией клинических состояний, способных значимо повлиять на результаты исследования. Характеристика пациентов представлена в табл. 1. По основным перечисленным характеристикам группы были относительно однородными.

У всех пациентов исследован уровень маркеров воспаления: СРБ (референсные значения: 0-3 мг/л) и фер- 
Таблица 1. Характеристика наблюдавшихся пациентов

\begin{tabular}{|l|c|c|}
\hline \multicolumn{1}{|c|}{ Показатель } & Основная группа & Контрольная группа \\
\hline Количество пациентов, $n$ & 70 & 60 \\
\hline Возраст, годы & $57,0 \pm 6,9[38 ; 65]$ & $53,6 \pm 6,6[42 ; 65]$ \\
\hline Пол пациентов (жен./муж.), \% & $61,1 / 38,9[43 ; 27]$ & $70 / 30[42 ; 18]$ \\
\hline СреАний койко-Аень, Ани & $13,7 \pm 3,3[7 ; 25]$ & $12,6 \pm 3,2[7 ; 22]$ \\
\hline
\end{tabular}

ритин (референсные значения: 10-150 нг/мл у женщин; 20-350 нг/мл у мужчин) минимум 2 раза за период стационарного лечения, при разнице показателей учитывали наибольший, а также уровень ИЛ-6 (при необходимости у 50 пациентов основной группы и 31 пациента из группы контроля, минимум 2 раза за период госпитализации, референсные значения: 0-10 пг/мл). Концентрацию СРБ в сыворотке и плазме крови определяли с помощью иммунотурбидиметрического метода (тест-система «СРБ-иммунотурбидиметрический ДД(»), концентрацию ферритина и ИЛ-6 с помощью иммуноферментного анализа с использованием тест-систем «Ферритин-ИФА-БЕСТ» и «Интерлейкин-6-ИФА-БЕСТ» соответственно. Пациенты обеих групп получали лечение, согласно Рекомендациям, действовавшим на момент госпитализации пациента [12]. В качестве антигипертензивной терапии использовали схему, к которой пациент был адаптирован до госпитализации. Все пациенты по окончании лечения выписаны с улучшением.

В связи с широким диапазоном изменений и наличием открытых значений лабораторных данных (>500 нг/мл) показателя ферритина при анализе использовали интервальный метод. Учитывая значительные гендерные различия референсных значений уровня ферритина и для удобства дальнейших расчетов при группировке переменные величины ферритина распределены на 11 интервалов с шагом в 50 нг/мл: <50 нг/мл, 51-100, 101-150... 451-500, $>500$ нг/мл.

Статистическая обработка данных проведена с помощью программы IBM SPSS Statistics 25.0. Определяли нормаль- ность распределения данных с применением критерия Колмогорова-Смирнова. В случаях, не подчиняющихся закону нормального распределения, использовали непараметрический критерий Манна-Уитни для оценки различий в 2 несвязанных выборках. При нормальном распределении признака использовали $t$-критерий Стьюдента. Различия считали достоверными при $p<0,05$.

\section{Результаты и обсужление}

Сравнение уровня ферритина в 2 группах показало, что выбранный конечный ориентир его превышения (>500 нг/мл) был выявлен у пациентов обеих групп примерно с одинаковой частотой (30\% в основной группе и 23,3\% - контрольной). Однако превышение средних уровней ферритина в основной группе было достоверно выше аналогичных показателей контрольной группы $(p=0,033 ; p<0,05)$.

При анализе с учетом гендерного фактора установлена разница между уровнем ферритина у мужчин и женщин в сравниваемых группах. Практически у $1 / 3$ (30,3\%) женщин основной группы уровень ферритина находился на уровне 251-350 нг/мл, в то время как у женщин контрольной группы, наоборот, данный уровень определили всего у 7,1\% пациенток. Показатель ферритина >350 нг/мл выявлен практически у одинакового числа женщин основной и контрольной групп (44,2 и 43\% соответственно). Выявленные различия с учетом интервального диапазона имеет значение, поскольку, согласно Рекомендациям, уровень ферритина $>250$ нг/мл при наличии признаков пневмонии (стадия КТ 1-4) и еще одного критерия является одним из показаний к назначению

Таблица 2. Распределение показателей ферритина сыворотки крови у пациентов основной и контрольной группы в зависимости от пола

\begin{tabular}{|c|c|c|c|c|c|c|c|c|c|c|c|c|}
\hline \multirow{3}{*}{$\begin{array}{c}\text { Уровень } \\
\text { ферритина, нг/м^ }\end{array}$} & \multicolumn{6}{|c|}{ Основная группа } & \multicolumn{6}{|c|}{ Контрольная группа } \\
\hline & \multicolumn{2}{|c|}{ общее значение } & \multicolumn{2}{|c|}{ женщины } & \multicolumn{2}{|c|}{ мужчины } & \multicolumn{2}{|c|}{ общее значение } & \multicolumn{2}{|c|}{ женщины } & \multicolumn{2}{|c|}{ мужчины } \\
\hline & aбc. & $\%$ & aбc. & $\%$ & aбc. & $\%$ & a6c. & $\%$ & a6c. & $\%$ & aбc. & $\%$ \\
\hline $0-50$ & 0 & 0,0 & 0 & 0,0 & 0 & 0,0 & 3 & 5,0 & 3 & 7,1 & 0 & 0,0 \\
\hline $51-100$ & 2 & 2,8 & 2 & 4,6 & 0 & 0,0 & 5 & 8,3 & 4 & 9,5 & 1 & 5,6 \\
\hline $101-150$ & 3 & 4,3 & 2 & 4,6 & 1 & 3,7 & 6 & 10,0 & 6 & 14,3 & 0 & 0,0 \\
\hline $151-200$ & 6 & 8,6 & 4 & 9,3 & 2 & 7,4 & 4 & 6,7 & 4 & 9,5 & 0 & 0,0 \\
\hline $201-250$ & 3 & 4,3 & 3 & 7,0 & 0 & 0,0 & 6 & 10,0 & 4 & 9,5 & 2 & 11,1 \\
\hline $251-300$ & 6 & 8,6 & 6 & 14,0 & 0 & 0,0 & 3 & 5,0 & 0 & 0,0 & 3 & 16,6 \\
\hline $301-350$ & 10 & 14,3 & 7 & 16,3 & 3 & 11,1 & 6 & 10,0 & 3 & 7,1 & 3 & 16,6 \\
\hline $351-400$ & 3 & 4,3 & 2 & 4,6 & 1 & 3,7 & 3 & 5,0 & 2 & 4,9 & 1 & 5,6 \\
\hline $401-450$ & 5 & 7,1 & 3 & 7,0 & 2 & 7,4 & 6 & 10,0 & 4 & 9,5 & 2 & 11,1 \\
\hline $451-500$ & 11 & 15,7 & 7 & 16,3 & 4 & 14,8 & 4 & 6,7 & 3 & 7,1 & 1 & 5,6 \\
\hline$>500$ & 21 & 30,0 & 7 & 16,3 & 14 & 51,9 & 14 & 23,3 & 9 & 21,5 & 5 & 27,8 \\
\hline Итого & 70 & 100 & 43 & 100 & 27 & 100 & 60 & 100 & 42 & 100 & 18 & 100 \\
\hline
\end{tabular}

Примечание. Зеленый цвет - нормальные значения ферритина; желтый цвет - умеренное повышение уровня ферритина; красный цвет - выраженное повышение уровня ферритина. 

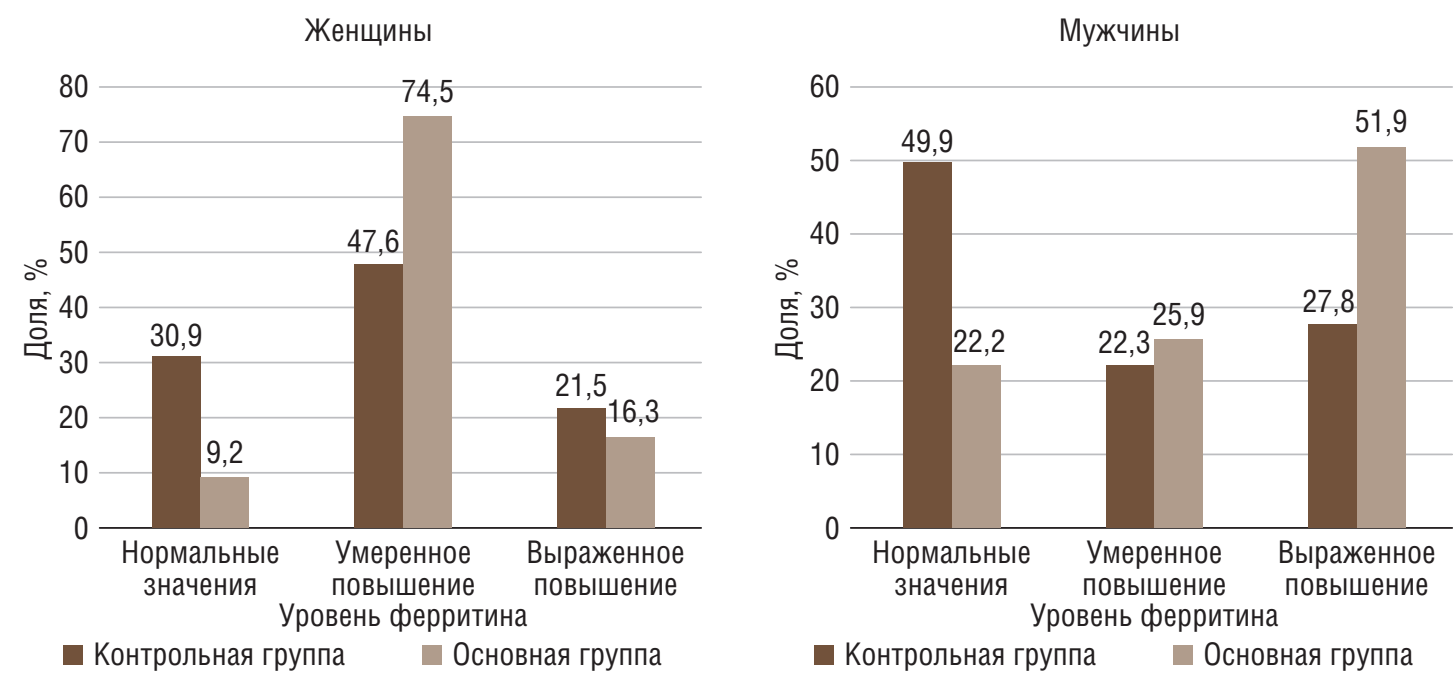

Различия уровней ферритина у пациентов основной и контрольной групп в зависимости от гендерного фактора

ингибитора рецепторов ИЛ-6 или ИЛ-1 $\beta$ [12]. Уровень ферритина $>250$ нг/мл определен у 74,5\% пациенток основной группы и у 50,1\% пациенток контрольной группы $(p=0,042)$. Следовательно, пациентки с COVID-19 на фоне ГБ чаще нуждаются в назначении этих биологических препаратов.

Содержание ферритина в сыворотке крови у мужчин основной группы было достоверно выше по сравнению с мужчинами контрольной группы $(p=0,048 ; p<0,05)$. Уровень ферритина выше 250 нг/мл определяли у 88,9\% мужчин основной группы и 83,3\% мужчин контрольной группы $(p=0,061)$. Причем профиль распределения показателей уровня ферритина значительно отличался от профиля у женщин и больше напоминал распределение показателей ферритина в целом по группе наблюдения. Следует отметить, что доля мужчин в группах значительно уступала доле женщин.

В основной группе среди мужчин в 66,7\% случаев выявлен уровень ферритина >451 нг/мл, в то время как в контрольной группе превышение данного уровня определено в $33,4 \%$ случаев $(p=0,039)$. В табл. 2 приведены профили распределения показателя уровня ферритина в основной и контрольной группах с учетом гендерного фактора.

В связи с тем что у женщин показатели ферритина $>151$ нг/мл соответствуют нормальным значениям ферритина у мужчин в данном диапазоне из-за значительной разницы референсных значений по гендерному признаку, было решено разделить показатели ферритинемии на 3 группы: нормальный уровень ферритина (10-150 нг/мл у женщин и 20-350 нг/мл у мужчин), умеренное (151-500 нг/мл у женщин и 351-500 нг/мл у мужчин) и выраженное повышение (>500 нг/мл). При анализе полученных данных установлено, что гиперферрринемия значительно чаще наблюдается в группе пациентов с сопутствующей ГБ, чем в контрольной группе $(p=0,017 ; p<0,05)$.

Нормальные значения ферритина выявлены лишь у незначительного числа пациенток (9,2\%), страдающих ГБ (основная группа), в то время как у женщин контрольной группы его уровень не превышал нормы в 30,9\% случаев $(p=0,045)$. Чаще всего умеренную степень гиперферритинемии наблюдали у пациенток обеих групп с ее преобладанием в основной группе (74,5 против 47,6\% в группе контроля, $p=0,031)$. Выраженная степень гиперферритинемии установлена примерно в одинаковом количестве случаев $(16,3 \%$ женщин основной группы и 21,5\% - контрольной).

Среди мужчин гиперферритинемия установлена у 77,8\% пациентов основной группы и у 50,1\% в контрольной $(p=0,023)$. Умеренное повышение ферритина выявлено у $25,9 \%$, а выраженное - у 51,9\% мужчин основной группы, в то время как в контрольной группе доля случаев умеренного и выраженного повышения составила 22,3 и 27,8\% соответственно. Выраженное повышение ферритина (>500 нг/мл) чаще зарегистрировано у пациентов мужского пола с COVID-19 на фоне сопутствующей ГБ $(p=0,036)$. На рисунке представлены значения уровней ферритина у пациентов основной и контрольной групп в зависимости от гендерного фактора.

На основании полученных данных можно предположить, что у женщин c COVID-19 на фоне ГБ преимущественно выявлено повышение уровня ферритина до умеренных значений, а у мужчин чаще определялась выраженная гиперферритинемия.

СРБ - наиболее известный и доступный маркер острого воспаления, он также чаще был повышен у пациентов с инфекцией, вызванной SARS-CoV-2, протекающей на фоне ГБ II стадии (с поражением органов-мишеней), чем у пациентов

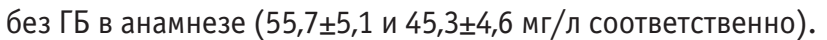

Среднее значение ИЛ-6 в обеих группах находилось

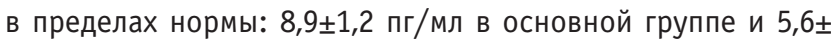
1,1 пг/мл в контрольной группе. 0днако у 17 (34\%) пациентов основной группы наблюдали повышение уровня ИЛ-6 более референсных значений (до 10 пг/мл), из них у 11 (22\%) пациентов - до 2 норм, у 6 (12\%) - до 3 норм. В контрольной группе повышение ИЛ-6 более референсных значений определили у $5(16,1 \%)$ пациентов $(p=0,129)$, из них у 4 (12,9\%) - двукратное, а у 1 (3,2\%) пациента - троекратное превышение нормальных значений. Согласно Рекомендациям, назначение иммунобиологических препаратов требуется при повышении уровня ИЛ-6 >40 пг/мл, однако данный уровень гиперферментемии является показателем «цитокинового шторма» и, соответственно, критерием исключения пациентов из исследования. 
Комплексная оценка маркеров воспаления (ферритин, ИЛ-6 и (РБ) позволяет предполагать наличие взаимосвязи ГБ с выраженностью ответной воспалительной реакции организма при COVID-19. Основанием для данной взаимосвязи могут быть несколько факторов, и важнейшим из них является высокая экспрессия ангиотензин-превращающего фермента II типа, который SARS-CoV-2 использует для проникновения в клетки-мишени в сосудистом эндотелии, в результате чего происходит прямое поражение сосудов, которые подвержены сосудистой (эндотелиальной) дисфункции при артериальной гипертонии [13-15]. Именно эндотелиальная дисфункция может быть одной из причин тяжести течения COVID-19, частого возникновения системных васкулитов, так как при функциональном изменении структуры эндотелия в условиях повышенной нагрузки на сосуды при ГБ происходит высвобождение провоспалительных цитокинов [15], что обусловливает необходимость изучения уровня маркеров острого воспаления при АГ. Несомненно, данный процесс не может быть главенствующим, однако он способен существенно повлиять на течение
COVID-19 и играть значимую роль в патогенезе осложнений инфекции, особенно со стороны сердечно-сосудистой системы.

\section{Зак^ючение}

У пациентов c COVID-19, протекающей на фоне ГБ, достоверно возрастают уровни ряда маркеров острого воспаления (ферритин, ИЛ-6) по сравнению с пациентами без сопутствующей АГ. У женщин преобладает умеренная степень повышения уровня ферритина (151-500 нг/мл), а у мужчин наблюдается выраженное повышение (>500 нг/мл). Пациентам с гиперферритинемией может потребоваться патогенетическая терапия иммунобиологическими препаратами (например, ингибиторами рецепторов ИЛ-6), однако степень АГ может стать препятствием для их использования. Согласно Рекомендациям, ГБ является противопоказанием для назначения терапии биологическими препаратами и требуется тщательно взвешивать риски и ожидаемую эффективность от их назначения.

\section{CBEAEHИЯ ОБ АВTOPAX}

ФГБОУ ВО КГМУ Минздрава России, Курск, Российская Федерация:

Киселева Виктория Валентиновна (Victoria V. Kiseleva)* - кандидат медицинских наук, доцент, заведующий кафедрой инфекционных болезней и эпидемиологии

E-mail:vvkiseleva@icloud.com

https://orcid.org/0000-0002-4138-7673

Ячменев Кирилл Сергеевич (Kirill S. Yachmenev) - ординатор кафедры оториноларингологии

E-mail: abba.kirill@yandex.ru

https://orcid.org/0000-0002-5069-6259

Зайцева Людмила Юрьевна (Lyudmila Yu. Zaitseva) - кандидат медицинских наук, доцент кафедры педиатрии

E-mail: zajcevalu@kursksmu.net

https://orcid.org/0000-0002-0118-0846

\section{ヘИТЕРАТУРА}

1. Zhang T., Huang W.S., Guan W., Hong Z. et al. Risk factors and predictors associated with the severity of COVID-19 in China: a systematic review, meta-analysis, and meta-regression // J. Thorac. Dis. 2020. Vol. 12, N 12. P. 7429-7441. DOI: https://doi.org/10.21037/jtd-20-1743

2. Gao Y.D., Ding M., Dong X., Zhang J.J. et al. Risk factors for severe and critically ill COVID-19 patients: a review // Allergy. 2021. Vol. 76. P. 428-455. DOI: https://doi.org/10.1111/all.14657

3. Шляхто Е.В., Конради А.О, Арутюнов Г.П. и Ар. Руководство по Аиагностике и лечению болезней системы кровообращения в контексте пандемия COVID-19 // Российский кардиологический журнал. 2020. T. 25, № 3. C. 129-148. DOI: https://doi.org/10.15829/1560-4071-20203-3801

4. Kernan K.F., Carcillo J.A. Hyperferritinemia and inflammation // Int. Immunol. 2017. Vol. 29, N 9. P. 401-409. DOI: https://doi.org/10.1093/ intimm/dxx031

5. Cheng L., Li H., Li. L. et al. Ferritin in the coronavirus disease 2019 (COVID-19): a systematic review and meta-analysis // J. Clin. Lab. Anal. 2020. Vol. 34, N 10. Article ID e23618. DOI: https://doi.org/10.1002/ jcla. 23618

6. Singh M.K., Mobeen A., Chandra A., Joshi S. et al. A meta-analysis of comorbidities in COVID-19: which diseases increase the susceptibility of SARS-CoV-2 infection? // Comput. Biol. Med. 2021. Vol. 130. Article ID 104219. DOI: https://doi.org/10.1016/j.compbiomed.2021.104219

7. Du Y., Zhou N., Zha W., Lv Y. Hypertension is a clinically important risk factor for critical illness and mortality in COVID-19: a meta-analysis // Nutr. Metab. Cardiovasc. Dis. 2021. Vol. 31, N 3. P. 745-755. DOI: https:// doi.org/10.1016/j.numecd.2020.12.009

8. Huang S., Wang J., Liu F., Liu J. et al. COVID-19 patients with hypertension have more severe disease: a multicenter retrospective observational study // Hypertens. Res. 2020. Vol. 43, N 8. P. 824-831. DOl: https://doi.org/10.1038/s41440-020-0485-2

9. Mesas A.E., Cavero-Redondo I., Alvarez-Bueno C., Sarria Cabrera M.A. et al. Predictors of in-hospital COVID-19 mortality: a comprehensive systematic review and meta-analysis exploring differences by age, sex and health conditions // PLoS One. 2020. Vol. 15, N 11. Article ID e0241742. DOI: https://doi.org/10.1371/journal.pone.0241742

10. Colafrancesco S., Alessandri C., Conti F., Priori R. COVID-19 gone bad: a new character in the spectrum of the hyperferritinemic syndrome? // Autoimmun. Rev. 2020. Vol. 19, N 7. Article ID 102573. DOl: https://doi.org/10.1016/j.autrev.2020.102573

11. Feld J., Tremblay D., Thibaud S., Kessler A., Naymagon L. Ferritin levels in patients with COVID-19: a poor predictor of mortality and he-

\footnotetext{
* Автор для корреспонденции.
} 
mophagocytic lymphohistiocytosis // Int. J. Lab. Hematol. 2020. Vol. 42 N 46. P. 773-779. DOI: https://doi.org/10.1111/ijlh.13309

12. Временные методические рекомендации «Профилактика, Аиагностика и мечение новой коронавирусной инфекции (COVID-19). Версии 7-9 (утв. Министерством зАравоохранения РФ 3 июня 2020 (версия 7), 3 сентября 2020 (версия 8), 26 октября 2020 (версия 9)). URL: https:// minzdrav.gov.ru/ministry/med_covid19 (Аата обращения: 13.04.2021)

13. Zhang J., Tecson K.M., McCullough P.A. Endothelial dysfunction contributes to COVID-19-associated vascular inflammation and coagulopathy // Rev. Cardiovasc. Med. 2020. Vol. 21, N 3. P. 315-319. DOI: https:// doi.org/ 10.31083/j.rcm.2020.03.126
14. Горшунова Н.К., Медведев Н.В., Рахманова О.В. Роль изме нений окислительно-восстановительных реакций в патогенезе эндотелиальной Аисфункции разной степени тяжести при артериальной гипертонии // Курский научно-практический вестник "Человек и его зАоровье». 2018. № 2. С. 20-26. DOI: https://doi.org/10.21626/vestnik/ 2018-2/03

15. Evans P.C., Rainger G.E., Mason J.C., Guzik T.J. et al. Endothelial dysfunction in COVID-19: a position paper of the ESC Working Group for Atherosclerosis and Vascular Biology, and the ESC Council of Basic Cardiovascular Science // Cardiovasc. Res. 2020. Vol. 116, N 14. P. 2177-2184 DOI: https://doi.org/10.1093/cvr/cvaa230

\section{REFERENCES}

1. Zhang T., Huang W.S., Guan W., Hong Z., et al. Risk factors and predictors associated with the severity of COVID-19 in China: a systematic review, meta-analysis, and meta-regression. J Thorac Dis. 2020; 12 (12): 7429-41. DOI: https://doi.org/10.21037/jtd-20-1743

2. Gao Y.D., Ding M., Dong X., Zhang J.J., et al. Risk factors for severe and critically ill COVID-19 patients: a review. Allergy. 2021; 76: 428-55. DOI: https://doi.org/10.1111/all.14657

3. Shlyakhto E.V., Konradi A.O., Arutyunov G.P., et al. Guidelines for the diagnosis and treatment of circulatory diseases in the contex of the COVID-19 pandemic. Rossiyskiy kardiologicheskiy zhurnal [Russian Journal of Cardiology]. 2020; 25 (3): 129-48. DOI: https://doi. org/10.15829/1560-4071-2020-3-3801 (in Russian)

4. Kernan K.F., Carcillo J.A. Hyperferritinemia and inflammation. Int Immunol. 2017; 29 (9): 401-9. DOI: https://doi.org/10.1093/intimm/dxx031

5. Cheng L., Li H., Li. L., et al. Ferritin in the coronavirus disease 2019 (COVID-19): a systematic review and meta-analysis. J Clin Lab Anal. 2020; 34 (10): e23618. DOI: https://doi.org/10.1002/jcla.23618

6. Singh M.K., Mobeen A., Chandra A., Joshi S., et al. A meta-analysis of comorbidities in COVID-19: which diseases increase the susceptibility of SARS-CoV-2 infection? Comput Biol Med. 2021; 130: 104219. DOI: https:// doi.org/10.1016/j.compbiomed.2021.104219

7. Du Y., Zhou N., Zha W., Lv Y. Hypertension is a clinically important risk factor for critical illness and mortality in COVID-19: a meta-analysis. Nutr Metab Cardiovasc Dis. 2021; 31 (3): 745-55. DOI: https://doi. org/10.1016/j.numecd.2020.12.009

8. Huang S., Wang J., Liu F., Liu J., et al. COVID-19 patients with hypertension have more severe disease: a multicenter retrospective observational study. Hypertens Res. 2020; 43 (8): 824-31. DOI: https://doi. org/10.1038/s41440-020-0485-2

9. Mesas A.E., Cavero-Redondo I., Alvarez-Bueno C., Sarria Cab rera M.A., et al. Predictors of in-hospital COVID-19 mortality: a comprehen- sive systematic review and meta-analysis exploring differences by age, sex and health conditions. PLoS One. 2020; 15 (11): e0241742. DOI: https:// doi.org/10.1371/journal.pone.0241742

10. Colafrancesco S., Alessandri C., Conti F., Priori R. COVID-19 gone bad: a new character in the spectrum of the hyperferritinemic syndrome? Autoimmun Rev. 2020; 19 (7): 102573. DOI: https://doi.org/10.1016/j autrev.2020.102573

11. Feld J., Tremblay D., Thibaud S., Kessler A., Naymagon L. Ferritin levels in patients with COVID-19: a poor predictor of mortality and hemophagocytic lymphohistiocytosis. Int J Lab Hematol. 2020; 42 (46): 773-9. DOI: https://doi.org/10.1111/ijlh.13309

12. Temporary guidelines "Prevention, diagnosis and treatment of new coronavirus infection (COVID-19). Versions 7-9" (approved by the Ministry of Health of the Russian Federation on June 3, 2020 (version 7), September 3 , 2020 (version 8), October 26, 2020 (version 9)). URL: https://minzdrav. gov.ru/ministry/med covid19 (date of access April 13, 2021) (in Russian)

13. Zhang J., Tecson K.M., McCullough P.A. Endothelial dysfunction contributes to COVID-19-associated vascular inflammation and coagulopathy. Rev Cardiovasc Med. 2020; 21 (3): 315-9. D0I: https://doi.org/ 10.31083/j.rcm.2020.03.126

14. Gorshunova N.K., Medvedev N.V., Rakhmanova O.V. Role of oxidative reactions changes in the pathogenesis of endothelial dysfunction of different severity in arterial hypertension. Kurskiy nauchno-prakticheskiy vestnik "Chelovek i ego zdorov'e" [Kursk Scientific and Practical Bulletin "Man and His Health"]. 2018; (2): 20-26. DOI: https://doi.org/10.21626/ vestnik/2018-2/03 (in Russian)

15. Evans P.C., Rainger G.E., Mason J.C., Guzik T.J., et al. Endothelia dysfunction in COVID-19: a position paper of the ESC Working Group for Atherosclerosis and Vascular Biology, and the ESC Council of Basic Cardiovascular Science. Cardiovasc Res. 2020; 116 (14): 2177-184. DOI: https:// doi.org/10.1093/cvr/cvaa230 